(2) Open Access Full Text Article

\title{
Improved Efficacy of Topical Latanoprost $0.005 \%$ Demonstrated by Corneal Biomechanical Correcting Modified Goldmann Prism
}

This article was published in the following Dove Press journal: Clinical Ophthalmology

\author{
Nathan Radcliffe (D) \\ John Berdahl (iD) ${ }^{2}$ \\ Mitchel Ibach ${ }^{2}$ \\ Justin Schweitzer ${ }^{2}$ \\ Jason Levine ${ }^{3}$ \\ Sean McCafferty ${ }^{3}$ \\ 'New York Eye Surgery Center, \\ New York, NY, USA; ${ }^{2}$ Vance Thompson \\ Vision, Sioux Falls, SD, USA; ${ }^{3}$ Arizona Eye \\ Consultants, Tucson, AZ, USA
}

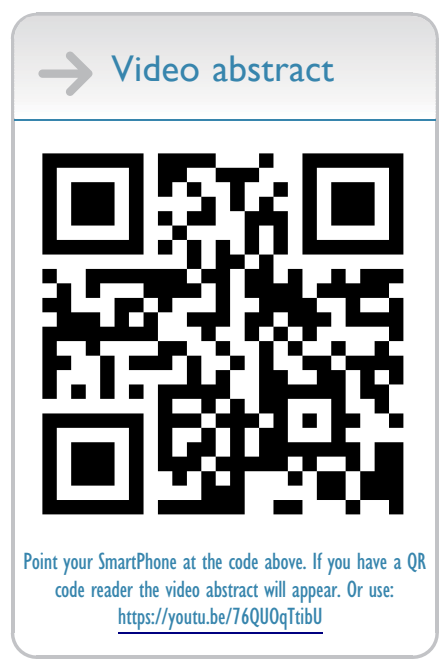

Correspondence: Sean McCafferty

Arizona Eye Consultants, 6422

E. Speedway Blvd, Tucson, AZ 857I0,

USA

Tel + I (520) 327-3487

Email SJMccafferty66@hotmail.com
Purpose: To evaluate intraocular pressure (IOP) reduction measured by a Goldmann applanation tonometer (GAT) prism and a modified surface Goldmann (CATS) prism with the institution of a topical prostaglandin analog (PGA) or alternatively a topical beta blocker.

Design: Prospective, open-label, randomized, controlled, and reference device comparison. Methods: Thirty-six (36) treatment naïve glaucoma patients (72 eyes) were randomized equally to treatment with latanoprost $0.005 \%$ or timolol maleate $0.5 \%$. Each patient underwent IOP measurement with standard GAT and CATS prisms before and at 1, 3, and 6 months of treatment. Central corneal thickness (CCT) and corneal hysteresis $(\mathrm{CH})$ were also measured. Medication response was defined as a $20 \%$ reduction in IOP from baseline.

Results: The CATS prism demonstrated the IOP reduction with topical latanoprost at a mean of $1.9 \mathrm{mmHg}$ lower than the IOP measured with GAT $(\mathrm{p}=0.01)$. The CATS and GAT prisms detected no difference in IOP reduction with timolol $(\mathrm{p}=0.23)$. The number of latanoprost treatment nonresponders was reduced from $36.1 \%$ measured with GAT to $13.8 \%$ when measured with the CATS prism $(\mathrm{p}=0.005)$. Timolol indicated no difference in the treatment non-response rate at $22.2 \%(p=0.999)$. $\mathrm{CH}$ increased significantly with latanoprost treatment by an average of 0.55 $\mathrm{mmHg}(\mathrm{p}=0.014)$ and remained unchanged with timolol at $-0.014 \mathrm{mmHg}(\mathrm{p}=0.68)$.

Discussion: IOP reduction and responder rates were increased when measured with a CATS prism in patients using latanoprost and not with timolol use. Latanoprost-induced alterations in corneal biomechanics may dampen the actual IOP reduction measured with a standard GAT prism.

Clinical Trial Registration: ClinicalTrials.gov NCT04178863.

Keywords: glaucoma, prostaglandins, IOP, tonometer, corneal biomechanics, timolol, latanoprost

\section{Plain Language Summary}

Latanoprost $0.005 \%$ drops for glaucoma may be more effective at lowering eye pressure by measurement with an improved accuracy tonometer device when compared to the historical standard tonometer device.

\section{Synopsis}

Latanoprost $0.005 \%$ reduction in IOP measured using a corneal biomechanical correcting Goldmann prism indicates $1.9 \mathrm{mmHg}$ lower IOP compared to that measured by a standard Goldmann prism, which is not seen with use of timolol $0.5 \%$. 


\section{Introduction}

Intraocular pressure (IOP) is the most important risk factor for glaucoma and glaucoma progression. ${ }^{1}$ It remains the only leading indicator of glaucoma progression and the primary modifiable parameter in the treatment of glaucoma. ${ }^{2}$ Goldmann Applanation Tonometry (GAT) remains the standard of care for IOP measurement. ${ }^{3}$ Variability in a patient's central corneal thickness (CCT), modulus of elasticity, corneal curvature, and tear film produces significant errors in GAT IOP measurement. ${ }^{4-6}$

The corneal biomechanical measurements of corneal hysteresis $(\mathrm{CH})$ and $\mathrm{CCT}$ have been shown as independent risk factors for the diagnosis and progression of glaucoma. Thin CCT is associated with glaucomatous progression demonstrated by field loss despite equal pressures to those patient's with normal CCTs. ${ }^{7-10}$ Low corneal hysteresis is also associated with optic nerve and visual field damage in glaucoma. ${ }^{11-13}$ Although both $\mathrm{CCT}$ and $\mathrm{CH}$ are seen as independent factors for glaucoma, their association with inaccurate IOP measurement likely contributes to the increased risk of progression. ${ }^{11,14,15}$

Topical prostaglandin analog (PGA) medications are among the most commonly used glaucoma drops and are highly effective at reducing IOP. ${ }^{16}$ Prostaglandins have been shown to significantly affect the biomechanical properties of tendinous collagenous tissue. ${ }^{17}$ Several studies have shown an increase in $\mathrm{CH}$ and Corneal Resistance Factor (CRF) with the use of PGAs. ${ }^{16,18,19}$ One study indicated possibly an opposite response with an increase in $\mathrm{CH}$ and CRF upon cessation of PGAs. ${ }^{20}$ The Corvis ST corneal response was shown to have a possible decrease in corneal stiffness with PGA use but the results were without a definitive indication as to the global change in corneal biomechanics. ${ }^{21}$ Although the question of whether PGA medications cause global corneal softening may be a significant oversimplification of the process particularly as a predictor of its effect on tonometry. There is growing evidence that corneal deformation is affected by a redistributed "buckling stress" and its behavior during applanation is more complex and highly non-linear. ${ }^{22-24}$ Topical timolol maleate drops have not been shown to have an effect on corneal biomechanical properties. ${ }^{21}$ Changes in IOP have been shown to change the cornea's modulus of elasticity and CCT which would affect tonometric IOP accuracy. ${ }^{4,6,22}$

A modified Goldmann prism (CATS) was recently cleared for use by the United States Food and Drug
Administration (US FDA) to measure IOP. The modified prism incorporates a correcting applanation tonometry surface (CATS) to the GAT prism and can replace the GAT prism on any Goldmann type tonometer. (Figure 1) Several studies, including intracameral pressure comparisons, have shown the CATS prism to have significantly decreased sensitivity to variations in corneal biomechanical properties when compared to the GAT prism. ${ }^{22,25-29}$ The design differences were described in detail previously. ${ }^{22}$ Differences in IOP between the CATS and GAT measurements were strongly correlated with variations in corneal biomechanical properties such as CCT and $\mathrm{CH}^{22,25,27-29}$ The CATS prism demonstrates a more accurate IOP as it negates much of the tonometer force due to corneal biomechanical deformation measuring predominantly the IOP when compared to the GAT prism. Furthermore, there was no overall IOP bias demonstrated between the two prisms over a large standard population. $^{22,25-29}$ Since both prisms measure the same pressure for a cornea with nominal biomechanical properties, it is likely that the difference in CATS and GAT IOP is a direct measurement (in $\mathrm{mm} \mathrm{Hg}$ ) of those combined corneal biomechanical properties affecting its deformation. ${ }^{25,26}$

The present study was completed to evaluate the effect of PGAs on IOP measurement accuracy and measure changes in corneal biomechanical properties. In addition, the study participants were equally randomized to timolol maleate treatment to delineate the effects of lowering IOP on the corneal biomechanics and IOP differences. Study design complies with the International Standards Organization (ISO) 8612:2009.

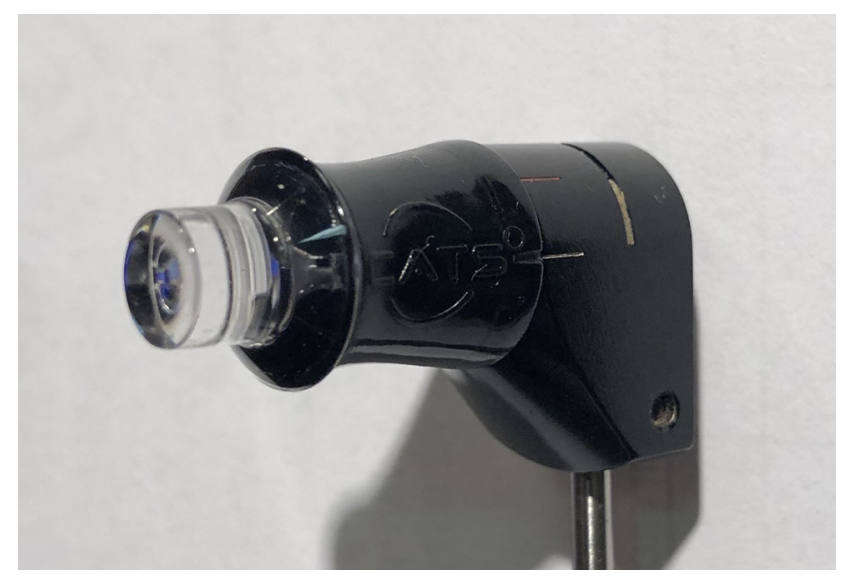

Figure I Applanating surface of the centrally concave and circumferentially convex (CATS) prism. 


\section{Methods}

Enrollment included treatment naïve glaucoma patients. Upon enrollment, subjects underwent a series of IOP measurements with the modified (CATS) and standard GAT tonometer prisms. The design was a randomized, controlled, prospective, open-labeled, device comparison. The study was registered prospectively on clinicaltrials. gov (NCT04178863) and IRB approved by Chesapeake. Eligible subjects were screened, enrolled, and evaluated according to the study protocol and were recruited from 2 sites. Participation in the study included subjects 18 years or older, meeting the protocol criteria, who provided written informed consent.

The clinical study was conducted within the ethical principles contained in Declaration of Helsinki, Code of Federal Regulations (CRF), Protection of Human Volunteers (21 CFR 50), Obligations of Clinical Investigators (21 CFR 812), and Institutional Review Boards (21 CFR 56)

\section{Description of Study Population}

Naïve glaucoma patients were enrolled into the study to include thin $(<600 \mu \mathrm{m})$ and normal corneas $(600$ $\mu \mathrm{m}>\mathrm{CCT}>500 \mu \mathrm{m})$. The 72 eye sample size was calculated using the difference between two paired means with an $\alpha=0.05$ (2-tailed), $\beta=0.2$, power $=0.85$ and the mean differences and standard deviations from previous studies. ${ }^{20,22,25}$

The following exclusionary conditions were prevented from study participation: corneal scarring, lid, corneal, or ocular conditions, disease, disorders, or infection that potentially affect corneal biomechanics and may have confounded the study results. Also excluded from the study were high myopes ( $>6$ diopters) and high astigmatism ( $>3$ diopters). Pregnant or nursing women and contact lens wearers were also excluded as well as any prior ocular surgery.

\section{Protocol}

Enrolled subjects received an ophthalmic exam from one of the investigators which included a new diagnosis of primary open-angle glaucoma by at least one of the following criteria: (1) visual field progression in at least one eye; (2) optic disc progression in at least one eye by OCT; (3) optic disc hemorrhages in at least 1 eye. ${ }^{30}$ Subjects were randomized by (a) random number generator to topical drop treatment with latanoprost $0.005 \%$ once daily or timolol maleate $0.5 \%$ twice daily. An Ocular Response Analyzer (ORA) was used to measure corneal hysteresis $(\mathrm{CH})$ and IOP (Reichert, Inc,
Depew, NY), by an assistant investigator. Central corneal thickness (CCT) was measured with a Zeiss HD-OCT-5000 spectral domain ocular coherence tomographer (Zeiss, Jena, Germany), by an assistant investigator.

Two Investigators measuring IOP were masked to the results of the assistant investigator's tests. Randomized initial use of the CATS and GAT prism devices was chosen by random number generator. Topical anesthetic drops with Fluorescein (fluorescein sodium and benoxinate hydrochloride ophthalmic solution $0.25 \% / 0.4 \%$, Bausch \& Lomb, Tampa, FL) were used before each measurement. A calibrated Haag-Streit model 900 applanation tonometer (Mason, $\mathrm{OH}$ ) was used to measure IOP with an alternated Haag-Streit GAT prisms and a CATS prism. Pressure measurements spaced by 5 minutes were made two (2) times with each a CATS and GAT prism (consisting of averaged measurements at 180 and 90 degrees to correct for astigmatism). ${ }^{26}$ Measurements were completed before treatment and at 1, 3, and 6 months following the institution of topical medication.

\section{Endpoints}

The primary endpoint was an IOP measurement comparison between modified (CATS) and GAT prisms with the use of latanoprost and timolol topical treatment for glaucoma. Also examining the difference between CATS and GAT IOP measurements' correlation to the known corneal biomechanical parameters of $\mathrm{CCT}$ and $\mathrm{CH}$ with the use of latanoprost and the use of timolol as a pressure lowering control. Nonresponse rates to the topical medications were defined as failure to lower the pressure by $20 \%$ from baseline IOP at 6 months and were calculated for both CATS and GAT prism use each with latanoprost and timolol. Validating analyses were completed on the changes seen in $\mathrm{CCT}$ and $\mathrm{CH}$ with the use of latanoprost and timolol.

\section{Statistical Methods}

The Full Analysis Set (FAS) was used to analyze the primary and secondary endpoints. The FAS included all eligible eyes.

Continuous variables were used for descriptive statistics including mean, standard deviation, median, and range. The primary endpoint was analyzed using a homoscedastic or paired, 2-tailed $t$-test $(\alpha=0.05)$. A linear regression analysis of the difference in paired GAT and CATS IOP measurements was correlated to $\mathrm{CCT}$ and $\mathrm{CH}$. A multiple linear regression analysis was 
Table I Table of Unique Patient and Eye Demographics in Test and Control Arms

\begin{tabular}{|l|l|l|l|}
\hline Indiv. Measurements & Total & Latanoprost $\mathbf{0 . 0 0 5 \%}$ & Timolol $\mathbf{0 . 5 \%}$ \\
\hline Unique eyes & 36 pts./72 eyes & 36 eyes from I8 pts. & 36 eyes from I8 pts. \\
Male,Female & 8,28 & 3,15 & 5,13 \\
Age \pm S.D. & $61 \pm 14$ & $60 \pm 15$ & $63 \pm 15$ \\
Initial CCT $(\mu \mathrm{m}) \pm$ S.D. & $540 \pm 34$ & $540 \pm 36$ & $541 \pm 33$ \\
Initial CH $(\mathrm{mmHg}) \pm$ S.D. & $9.7 \pm 1.3$ & $9.6 \pm 1.5$ & $9.8 \pm 0.95$ \\
Initial GAT IOP $(\mathrm{mmHg}) \pm$ S.D. & $20.7 \pm 7.0$ & $20.6 \pm 7.9$ & $20.8 \pm 6.2$ \\
\% by GAT/CATS initially below $21 \mathrm{mmHg}$ & $61.1 / 38.9$ & $63.9 / 33.3$ & $58.3 / 44.0$ \\
\hline
\end{tabular}

completed on the FAS using a general linear mixed effects model examining $\mathrm{CH}$, CCT, IOP, age, and gender.

\section{Results}

Seventy-two (72) eyes were measured from 36 patients. There were thirty-six (36) eyes enrolled from 18 patients with the use of latanoprost and thirty-six (36) eyes from 18 patients with the use of timolol. Four (4) eyes ( 2 patients) in the latanoprost group and six (6) eyes (3 patients) in the timolol group did not complete all measurements and were included in the FAS to the degree which they were completed. There were $3(17 \%)$ males and $15(83 \%)$ females with an average age of $60 \pm 15$ years who were enrolled in the latanoprost arm. There were $5(27 \%)$ males and $13(73 \%)$ females with an average age of $63 \pm 15$ years who were enrolled in the timolol control arm. CCT, CH, Initial IOP, and percent of eyes below $21 \mathrm{mmHg}$ were closely matched between cohorts (Table 1)

\section{CATS and GAT IOP Reduction Analysis}

The IOP reduction with both CATS and GAT measurements in each cohort is demonstrated before and at prescribed follow-up times in Figure 2 and Table 2.

Table 2 illustrates the differential IOP means. Initial paired CAT minus GAT IOP differences indicated an average of $1.95 \pm 2.23 \mathrm{~mm} \mathrm{Hg}$ higher IOP with the CATS prism compared to the GAT prism. Twenty-two percent $(22 \%)$ of the patients initially classified as normal tension glaucoma by GAT IOP measurement less than $21 \mathrm{mmHg}$ would have been reclassified as primary open angle glaucoma by the higher CATS IOP measurements at 6 months, a representative

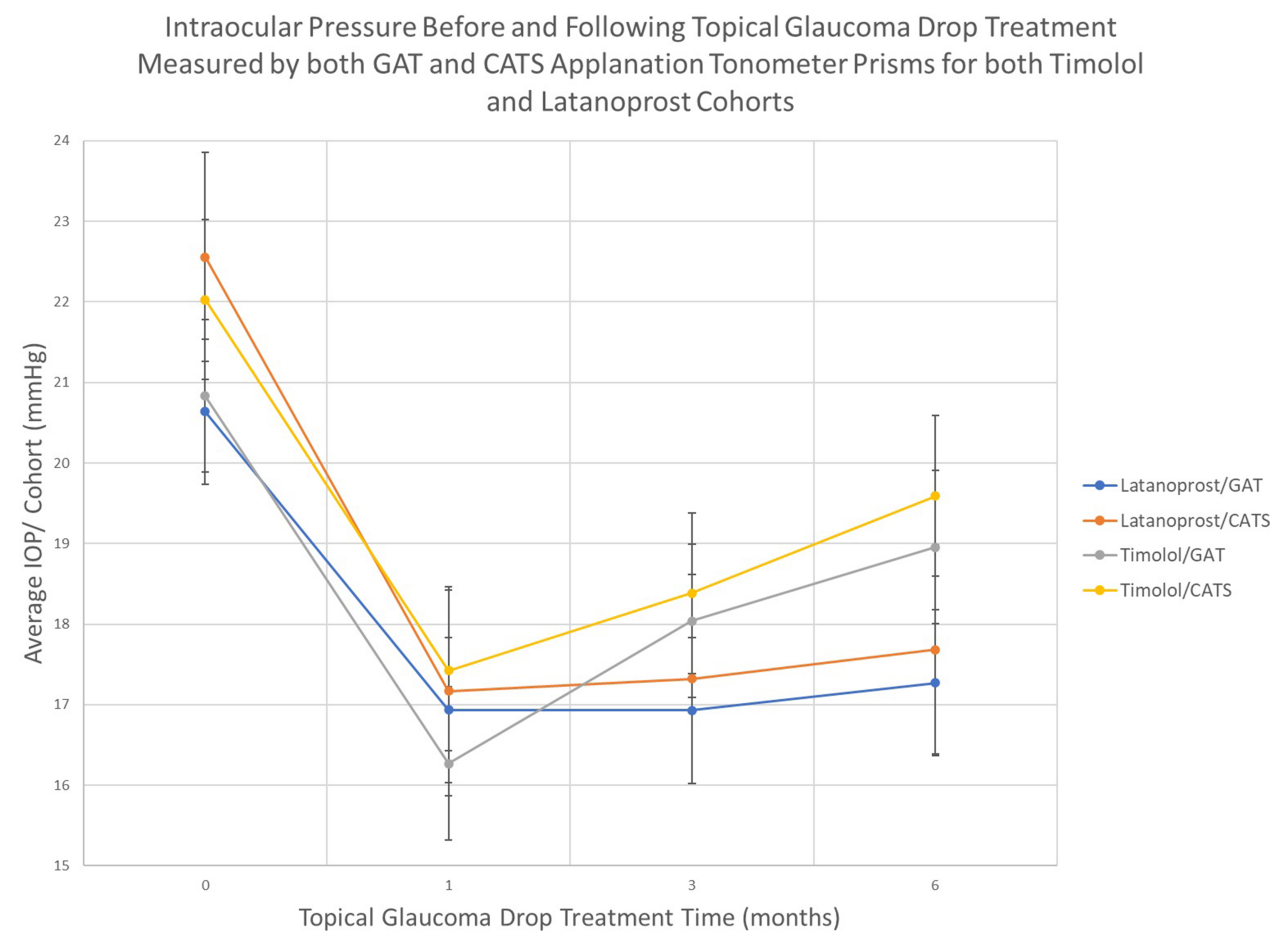

Figure 2 Timolol and latanoprost cohort IOP measurements at treatment times. 
Table 2 Timolol and Latanoprost Cohort Mean IOP Measurements and Standard Deviation at Treatment Examination Times

\begin{tabular}{|l|l|l|l|l|}
\hline & $\begin{array}{l}\text { Pre- } \\
\text { Treatment }\end{array}$ & $\begin{array}{l}\text { I } \\
\text { Month }\end{array}$ & $\begin{array}{l}\mathbf{3} \\
\text { Months }\end{array}$ & $\begin{array}{l}\mathbf{6} \\
\text { Months }\end{array}$ \\
\hline $\begin{array}{l}\text { Latanoprost cohort } \\
\text { Mean GAT }\end{array}$ & 20.64 & 16.93 & 16.93 & 17.27 \\
S.D. & 2.57 & 1.12 & 1.13 & 1.31 \\
Mean CATS & 22.56 & 17.17 & 17.32 & 17.68 \\
S.D. & 2.65 & 1.21 & 1.09 & 1.32 \\
CATS-GAT & 2.02 & 0.23 & 0.39 & 0.41 \\
Paired Difference & & & & \\
S.D. & 2.30 & 1.41 & 1.14 & 1.85 \\
\hline Timolol cohort & & & & \\
Mean GAT & 20.83 & 16.27 & 18.04 & 18.95 \\
S.D. & 2.03 & 1.37 & 1.64 & 1.90 \\
Mean CATS & 22.03 & 17.42 & 18.38 & 19.59 \\
S.D. & 2.11 & 1.27 & 1.49 & 1.69 \\
CATS-GAT Paired & 1.91 & 1.07 & 0.34 & 0.5 \\
Difference & & & & \\
S.D. & 2.02 & 1.77 & 1.54 & 1.5 \\
\hline
\end{tabular}

analysis of the differential IOP between paired GAT and CATS measurement is presented. The differential IOP at both the one and three month examination times is similar as shown in Figure 2 and Table 2. The IOP reduction with the CATS prism in patients using topical latanoprost $0.005 \%$ was normally distributed with an average of $6.5 \pm 5.9 \mathrm{mmHg}$ lower from baseline IOP at 6 months. The IOP reduction with the GAT prism in patients using latanoprost averaged $4.6 \pm 5.2 \mathrm{mmHg}$ lower from baseline at 6 months. The CATS prism IOP measurements were $1.9 \mathrm{mmHg}$ lower than the paired IOP measured with the GAT prism $(\mathrm{p}=0.01)$. (Table 3 )

CATS IOP reduction with timolol maleate $0.5 \%$ was $2.8 \pm 6.7 \mathrm{mmHg}$ compared to $3.3 \pm 6.7 \mathrm{mmHg}$ in the paired timolol control arm. The IOP measurements between CATS and GAT prisms did not demonstrate a significant difference with timolol $(\mathrm{p}=0.23)$. The GAT IOP reduction seen with latanoprost approached significantly lower than timolol in this sample size $(p=0.08)$. However, the IOP reduction of latanoprost compared to timolol was significantly lower when the IOP was measured with the CATS prism $(\mathrm{p}=0.008)$. (Table 3$)$

\section{CATS and GAT Non-Response Rate Analysis}

Topical treatment non-response was defined as a failure of the medication to lower the IOP by $20 \%$ from baseline at 6
Table 3 CATS and GAT IOP Reduction in Latanoprost $0.005 \%$ Test and Timolol 0.5\% Control Arms at 6 Months

\begin{tabular}{|c|c|c|c|}
\hline Prism & $\begin{array}{l}\text { Latanoprost } \\
0.005 \% \\
\mathrm{mmHg}\end{array}$ & $\begin{array}{l}\text { Timolol } \\
0.5 \% \\
\mathrm{mmHg}\end{array}$ & $\begin{array}{l}\text { Difference (Lat- } \\
\text { Tim) } \mathrm{mmHg} \\
\text { (p=homoscadastic) }\end{array}$ \\
\hline $\begin{array}{l}\text { CATS } \mathrm{mmHg} \pm \text { S.D. } \\
\text { GAT } \mathrm{mmHg} \pm \text { S.D. } \\
\text { IOP drop (Pre-6 } \\
\text { mos.) } \mathrm{mmHg} \\
\text { ( } \mathrm{p}=\text { paired) }\end{array}$ & $\begin{array}{l}6.5 \pm 5.9 \\
4.6 \pm 5.2 \\
1.9(p=0.01)\end{array}$ & $\begin{array}{l}2.8 \pm 6.7 \\
3.3 \pm 6.7 \\
-0.5 \\
(p=0.23)\end{array}$ & $\begin{array}{l}3.7(\mathrm{p}=0.008) \\
1.5(\mathrm{p}=0.08)\end{array}$ \\
\hline
\end{tabular}

months. The number of latanoprost treatment non-responders was reduced from $36.1 \%$ measured with the standard GAT prism to $13.8 \%$ when measured with the CATS prism $(\mathrm{p}=0.005)$. Timolol indicated no difference in the treatment non-response rate at $22.2 \%(p=0.999)$. (Figure 3 )

\section{Corneal Hysteresis and CCT Validation}

Corneal hysteresis $(\mathrm{CH})$ increased significantly with latanoprost treatment by an average of $0.55 \mathrm{mmHg}(\mathrm{p}=0.014)$ and remained unchanged with timolol at $-0.014 \mathrm{mmHg}$ $(\mathrm{p}=0.68)$. The subject's average $\mathrm{CH}$ was $9.6 \pm 1.5 \mathrm{mmHg}$ in the latanoprost arm and $9.8 \pm 0.95 \mathrm{mmHg}$ in the timolol control arm, before treatment.

Central corneal thickness (CCT) increased from baseline but not significantly by $0.5 \%(\mathrm{p}=0.27)$ in the latanoprost group and decreased by $0.9 \%$ in the timolol group $(p=0.10)$. However, the difference in CCT change between the latanoprost test and timolol control groups was statistically increased in the latanoprost group by $1.4 \%$ $(p=0.005)$.

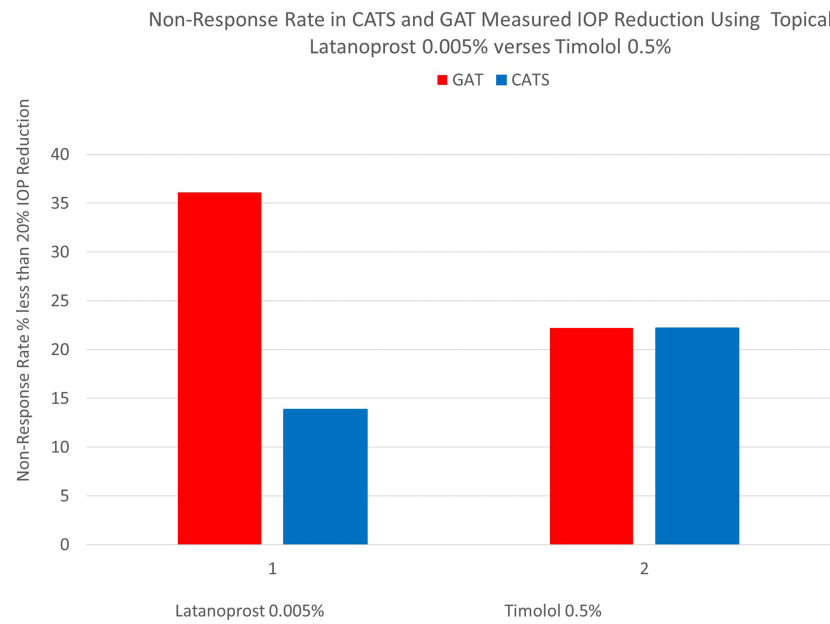

Figure 3 Non-response rates less than 20\% reduction from baseline IOP in CATS and GAT measured IOP reduction using topical latanoprost $0.005 \%$ or alternatively timolol $0.5 \%$. 
The general linear mixed effects (GLME) model indicated significant and nearly significant correlations between the difference in CATS and GAT IOP measurements to CCT and $\mathrm{CH}(\mathrm{p}=0.010, \mathrm{p}=0.065)$. Age also correlated to the difference in CATS and GAT IOP measurements to CCT and $\mathrm{CH}(\mathrm{p}=0.025, \mathrm{p}=0.016)$. No significant correlation was seen in either group or combined groups between the difference in CATS and GAT IOP measurements nor $\mathrm{CH}$ to IOP.

\section{Conclusions}

Significantly greater IOP reduction was demonstrated using a PGA, latanoprost $0.005 \%$, by IOP measurement with a corneal biomechanical correcting modified Goldmann (CATS) prism. No additional timolol 0.5\% IOP reduction was seen using the CATS prism with when compared to the standard flat applanation surface GAT prism. These results were demonstrated at 1.3, and 6 months. The GAT measured IOP reduction approached statistically lower with latanoprost compared to timolol cohorts. However, modified CATS IOP measurement shows the latanoprost group demonstrates significantly greater IOP reduction than the timolol group.

The modified CATS prism is reading a greater pressure reduction than has been previously demonstrated with PGAs measured by GAT. It is unlikely that these results indicate a global change in corneal rigidity with PGAs. The authors see evidence the study findings are consistent with the PGA inducing a stress redistribution within the stromal lamellae. The corneal response to applanation and the changes due to PGAs appear more complex than a simplified global metric of increased or decreased corneal stiffness is able to predict. Modeling used to design the modified applanating surface of the CATS prism indicated that the pressure across the applanation surface is not constant as is has been presumed. ${ }^{22}$ The local pressure force at the center of the applanation diameter approaches zero using a flat surfaced Goldmann prism and the cornea actually buckles or dimples centrally. ${ }^{15,22}$ This corneal buckling is supported by other studies. ${ }^{23,24}$ The modified CATS prism surface equalizes this pressure distribution across the applanation surface and demonstrates a significantly decreased sensitivity to alterations in the major sources of corneal biomechanical error. ${ }^{22,25-29}$

Decreased non-responder rates were also demonstrated with a modified Goldmann (CATS) prism in patients using the PGA latanoprost and not with timolol use. Part of the latanoprost non-response may be latanoprost induced alterations in corneal biomechanics which dampen the actual IOP reduction measured with a standard GAT prism.

Corneal hysteresis was shown to increase in the PGA latanoprost group and not in the timolol group. Hysteresis and corneal rigidity are parameters that tend to follow each other as they are related under specific narrow assumptions. However, $\mathrm{CH}$ is a dynamic global corneal dampening coefficient and not a static spring constant measuring global corneal stiffness or rigidity. The difference in CATS and GAT IOP differs from $\mathrm{CH}$ in that it is likely a measure of the static spring constant or average corneal rigidity at full applanation. The study findings corroborate well with previous studies and likely validate the effect of corneal stress redistribution and buckling previously discussed. ${ }^{16,18,19,23,24}$

CATS tonometer prism IOP measurements have been shown substantially equivalent to flat surfaced GAT prism IOP measurements in patients without disease such as glaucoma and having nominal CCTs between 500 and 600 microns. $^{26,27}$ Initial differential CATS minus GAT IOP measurements indicated an average of $1.95 \pm$ $2.23 \mathrm{~mm} \mathrm{Hg}$ higher IOP with the CATS prism compared to the GAT prism. Additionally, $22 \%$ of the patients would be reclassified as primary open-angle glaucoma from normal tension glaucoma based upon the higher IOP measurement with the CATS prism. The obvious difference accounting for the pre-treatment higher IOP bias with CATS is that these are patients with glaucoma and about half are normal tension glaucoma. The CATS prism has been shown to correct for corneal biomechanical errors in IOP and the difference between CATS and GAT is likely a measure of relative corneal biomechanical properties as demonstrated by its correlation to CCT and $\mathrm{CH} .{ }^{22,23,26-29}$ Similar measures of corneal biomechanical properties such as low $\mathrm{CH}$ have been shown to have a significant predictive value for glaucoma progression and higher prevalence in glaucoma patients including NTG patients. ${ }^{13,16}$ Similarly, this glaucoma pre-treatment bias suggests there may be higher predictive value for the diagnosis or progression of glaucoma with CATS IOP measurements using historical benchmarks such as $21 \mathrm{~mm} \mathrm{Hg}$ when compared to GAT prism IOP. A longitudinal study examining glaucoma diagnosis and progression would be required to confirm this hypothesis.

It is possible that latanoprost increased $\mathrm{CH}$ more than timolol because latanoprost is more effective at lowering IOP, particularly as it has been shown over the entire 24hour window. ${ }^{31}$ That selective laser trabeculoplasty (SLT) 
also increases $\mathrm{CH}$ in a manner similar to latanoprost, suggests that IOP lowering may itself elevate $\mathrm{CH}^{32}$ However no change in $\mathrm{CH}$ or CATS-GAT IOP difference was noted with IOP reduction in the Timolol group. Differential tonometry (using two different tonometers) has been described and used in several studies to measure changes in corneal rigidity. ${ }^{33}$ The differential tonometry between CATS and GAT IOP has an advantage in that the CATS prism was designed to have zero overall bias when compared to GAT. $^{26}$ However, whether latanoprost directly alters the corneal biomechanics or whether it elevates $\mathrm{CH}$ through its action on IOP, the magnitude of IOP lowering as measured by GAT measurements may remain underestimated.

Additional results include a statistically significant correlation between the CATS and GAT IOP measurement differences to CCT and nearly to $\mathrm{CH}$. This finding validates previous results demonstrating a decreased sensitivity in CATS IOP measurements to the corneal biomechanical parameters of CCT and $\mathrm{CH}^{22,25-29}$ The modified (CATS) and GAT prisms showed no differences in measurement based upon gender, or IOP, but indicated a statistically significant correlation with age. The agerelated increase in CATS-GAT IOP differences and related decrease in $\mathrm{CH}$ corroborate prior studies. ${ }^{9,15}$

Studies measuring IOP as a primary endpoint, such as glaucoma medication clinical trials, should include some correction for corneal biomechanical differences or alterations which create significant error in GAT. Even surgical studies that appear remote to the cornea can have a significant effect on corneal biomechanics and induce significant GAT error. ${ }^{34,35}$ Future studies include examining the difference in CATS-GAT IOP measurement differences before and after LASIK and corneal cross-linking as well as examining paired IOP differences pediatric populations.

\section{Abbreviations}

ANSI, American National Standards Institute; CATS, correcting applanation tonometry surface; CCT, Central Corneal Thickness; CH, corneal hysteresis; CFR, Code of Federal Regulations; CRF, corneal resistance factor; FDA, Food and Drug Administration; GAT, Goldmann applanation tonometer; IOP, intraocular pressure; IRB, Independent Review Board; ISO, International Standards Organization; NTG, normal tension glaucoma; ORA, Ocular Response Analyzer; PGA, prostaglandin analog; SLT, selective laser trabeculoplasty.

\section{Ethics and Consent to Participate}

This clinical study was conducted in accordance with the ethical principles contained within Declaration of Helsinki, Protection of Human Volunteers (21 CFR 50), Institutional Review Boards (21 CFR 56), and Obligations of Clinical Investigators (21 CFR 812). Deidentified data will be made available upon request to the corresponding author for a period of three years.

\section{Acknowledgments}

Arizona Eye Consultants, Tucson, AZ for extensive facilities use.

\section{Author Contributions}

All authors made a significant contribution to the work reported, whether that is in the conception, study design, execution, acquisition of data, analysis and interpretation, or in all these areas; took part in drafting, revising or critically reviewing the article; gave final approval of the version to be published; have agreed on the journal to which the article has been submitted; and agree to be accountable for all aspects of the work.

\section{Funding}

This study was supported in part by NIH SBIR Grant R43 EY026821-01 and Arizona Eye Consultants, Tucson, AZ.

\section{Disclosure}

Sean McCafferty has an interest in Intuor Technologies (Tucson, AZ) which owns the technology being examined in this clinical trial. Additional grant support unrelated to this study has been provided by Abbott Medical Optics (Santa Ana, CA), and Alcon, Inc. (Ft. Worth, TX). He also reports grants from NIH/NEI, during the conduct of the study; nonfinancial support from Reichert, outside the submitted work. Sean McCafferty has a patent on prism owned by CATS Tonometer issued. Jason Levine has unrelated study grant support from Innfocus, Inc. John Berdahl is a consultant to and reports personal fees from Intuor. Justin Schweitzer reports personal fees from Reichert, during the conduct of the study. Mitchel Ibach reports personal fees from Aerie pharmaceuticals, outside the submitted work. Nathan Radcliffe reports personal fees from Reichert, CATS, LLC, Allergan, Alcon, Novartis, Glaukos, Ivantis, New World Medical, Bausch \& Lomb, Omeros, Lumenis, and Ellex, outside the submitted work. The authors report no other conflicts of interest in this work. 


\section{References}

1. Heijl A, Leske MC, Hyman L, et al. Intraocular pressure reduction with a fixed treatment protocol in the Early Manifest Glaucoma Trial. Acta Ophthalmol. 2011;89:749-754. doi:10.1111/j.1755-3768.2009.01852.x

2. Chan M, Broadway D, Khawaja A, et al. Glaucoma and Intraocular pressure in EPIC-Norfolk Eye Study: cross sectional study. BMJ. 2017;358:3889. doi:10.1136/bmj.j3889

3. Ophthalmology AA. American academy of ophthalmology, preferred practice pattern; primary open-angle glaucoma. 2015. doi:10.1016/j. ophtha.2015.10.053

4. Liu J, Roberts C. Influence of cornea biomechanical properties on intraocular pressure measurement: quantitative analysis. $J$ Cat and Ref Surg. 2005;31:146-155. doi:10.1016/j.jcrs.2004.09.031

5. Damji K, Muni R, Munger R. Influence of corneal variables on accuracy of intraocular pressure measurement. $J$ Glaucoma. 2003;12:69-80. doi:10.1097/00061198-200302000-00015

6. Park S, Soon A, Nicholas S, Wells A. The Effect of thin, thick and normal corneas on Goldmann intraocular pressure measurements and correction formulae in individual eyes. Ophthalmology. 2012;119:443-449. doi:10.1016/j.ophtha.2011.07.058

7. Kass M, Heuer D, Higginbotham E, et al. The Ocular Hypertension Treatment Study: a randomized trial determines that topical ocular hypotensive medication delays or prevents the onset of primary open-angle glaucoma. Arch Ophthalmol. 2002;120:701-713. doi:10.1001/archopht.120.6.701

8. Iester M, Mete M, Figus M, Frezzotti P. Incorporating corneal Pachymetry into the management of glaucoma. $J$ Cataract Refract Surg. 2009;35(9):1623-1628. doi:10.1016/j.jcrs.2009.05.015

9. Kotecha A, Elsheikh A, Roberts C, Zhu H, Garway-Heath D. Corneal thickness- and age related biomechanical properties of the cornea measured with the ocular response analyzer. Invest Ophthalmol Vis Sci. 2006;47(12):5337-5347. doi:10.1167/iovs.06-0557

10. Medeiros F, Weinreb R. Is corneal thickness an independent risk factor for glaucoma? Ophthalmology. 2012;119:435-436. doi:10.10 16/j.ophtha.2012.01.018

11. Costin B, Fleming G, Weber P, Mahmoud A, Roberts C. Corneal biomechanical properties affect Goldmann applanation tonometry in primary open-angle glaucoma. $J$ Glaucoma. 2014;23:69-74. doi:10.1097/IJG.0b013e318269804b

12. Deol M, Taylor D, Radcliffe N. Corneal hysteresis and its relevance to glaucoma. Curr Opin Ophthalmol. 2015;26:96-102. doi:10.1097/ ICU.0000000000000130

13. Medeiros F, Meira-Freitas D, Lisboa R, Kuang T, Zangwill L, Weinreb R. Corneal hysteresis as a risk factor for glaucoma progression: a prospective longitudinal study. Ophthalmology. 2013;120 (8):1533-1540. doi:10.1016/j.ophtha.2013.01.032

14. Touboul D, Roberts C, Kerautret J, et al. Correlations between corneal hysteresis, intraocular pressure, and corneal central pachymetry. J Cataract Refract Surg. 2008;34:616-622. doi:10.10 16/j.jcrs.2007.11.051

15. McCafferty S, Levine J, Schwiegerling J, Enikov E. Goldmann applanation tonometry error relative to true intracameral Intraocular pressure in vitro and in vivo. BMC Ophthalmol. 2017;17:215. doi:10.1186/s12886-017-0608-y

16. Agarwal D, Ehrlich J, Shimmyo M. The relationship between corneal hysteresis and the magnitude of intraocular pressure reduction with topical prostaglandin therapy. $\mathrm{Br} J$ Ophthalmol. 2012;96:254-257. doi:10.1136/bjo.2010.196899

17. Ferry S, Afshari H, Lee J, Dahners L, Weinhold P. Effect of prostaglandin E2 injection on the structural properties of the rat patellar tendon. Sports Med Arthrosc Rehabil Ther Technol. 2012;4:2. doi:10.1186/1758-2555-4-2
18. Bolívar G, Sánchez-Barahona C, Teus M, et al. Effect of topical prostaglandin analogues on corneal hysteresis. Acta Ophthalmol. 2015;93:495-498. doi:10.1111/aos.12689

19. Tsikripis P, Papaconstantinou D, Koutsandrea C, Apostolopoulos M, Georgalas I. The effect of prostaglandin analogs on the biomechanical properties and central thickness of the cornea of patients with open-angle glaucoma: a 3-year study on 108 eyes. Drug Des Devel Ther. 2013;7:1149-1156. doi:10.2147/DDDT.S50622

20. Meda R, Wang Q, Paoloni D, Harasymowycz P, Brunette I. The impact of chronic use of prostaglandin analogues on the biomechanical properties of the cornea in patients with primary open-angle glaucoma. $B r \quad J$ Ophthalmol. 2017;101:120-125. doi:10.1136/ bjophthalmol-2016-308432

21. Amano S, Nejima R, Inoue K. Effect of topical prostaglandins on the biomechanics and shape of the cornea. Graefes Arch Clin Exp Ophthalmol. 2019;10:2213-2219.

22. McCafferty S, Lim G, Duncan W, Enikov E, Schwiegerling J. Goldmann tonometer prism with an optimized error correcting applanation surface. Transl Vis Sci Technol. 2016;5:1-5. doi:10.1167/ tvst.5.5.4

23. Jóźwik A, Kasprzak H, Kozakiewicz A. Corneal buckling during applanation and its effect on the air pressure curve in ocular response analyzer. Int $J$ Environ Res Public Health. 2019;16(15):2742. doi:10.3390/ijerph16152742

24. Asejczyk-Widlicka M, Srodka W. Finite element simulation of Goldmann tonometry after refractive surgery. Clinical Biomechanics. 2019;71:24-28.

25. McCafferty S, Tetrault K, McColgin A, Chue W, Levine J, Muller M. Modified Goldmann prism intraocular pressure measurement accuracy and correlation to corneal biomechanical metrics: multicentre randomised clinical trial. Br J Ophthal. 2019;103:1840-1844.

26. McCafferty S, Tetrault K, McColgin A, Chue W, Levine J, Muller M. Intraocular pressure measurement accuracy and repeatability of a modified Goldmann prism: multi-center randomized clinical trial. Am J of Ophthal. 2018;196:145-153. doi:10.1016/j.ajo.2018.08.051

27. McCafferty S, Lim G, Duncan W, Enikov E, Schwiegerling J. Goldmann tonometer error correcting prism: clinical evaluation. Clin Ophthalmol. 2017;11:835-840. doi:10.2147/OPTH.S135272

28. McCafferty S, Levine J, Schwiegerling J, Enikov ET. Goldmann and error correcting tonometry prisms compared to intracameral pressure. BMC Ophthalmol. 2018;18:1-9.

29. McCafferty S, Enikov E, Schwiegerling J, Ashley S. Goldmann tonometry tear-film error and partial correction with a shaped applanation surface. Clin Ophthal. 2018;12:71.

30. Öhnell H, Bengtsson B, Heijl A. Making a correct diagnosis of glaucoma: data from the EMGT. J Glaucoma. 2019;28:859-864. doi:10.1097/IJG.0000000000001342

31. Liu J, Kripke D, Weinreb R. Comparison of the nocturnal effects of once-daily timolol and latanoprost on intraocular pressure. $\mathrm{Am}$ J Ophthalmol. 2004;138:389-395. doi:10.1016/j.ajo.2004.04.022

32. Pillunat K, Spoerl E, Terai N, Pillunat L. Effect of selective laser trabeculoplasty on corneal biomechanics. Acta Ophthalmol. 2016;94:501-504. doi:10.1111/aos.12947

33. Detorakis E, Tsaglioti E, Kymionis G. Non-invasive ocular rigidity measurement: a differential tonometry approach. Acta Medica Cordoba. 2015;58:92-97. doi:10.14712/18059694.2015.99

34. Iordanidou V, Hamard P, Gendron G, Labbé A, Raphael M, Baudouin C. Modifications in corneal biomechanics and intraocular pressure after deep sclerectomy. J Glaucoma. 2010;19:252-256. doi:10.1097/IJG.0b013e3181aff419

35. Pakravan M, Afroozifar M, Yazdani S. Corneal biomechanical changes following trabeculectomy, phaco-trabeculectomy, ahmed glaucoma valve implantation and phacoemulsification. J Ophthalmic Vis Res. 2014;9:7-13. 


\section{Publish your work in this journal}

Clinical Ophthalmology is an international, peer-reviewed journal covering all subspecialties within ophthalmology. Key topics include: Optometry; Visual science; Pharmacology and drug therapy in eye diseases; Basic Sciences; Primary and Secondary eye care; Patient Safety and Quality of Care Improvements. This journal is indexed on PubMed

Submit your manuscript here: https://www.dovepress.com/clinical-ophthalmology-journal
Central and CAS, and is the official journal of The Society of Clinical Ophthalmology (SCO). The manuscript management system is completely online and includes a very quick and fair peer-review system, which is all easy to use. Visit http://www.dovepress.com/ testimonials.php to read real quotes from published authors. 